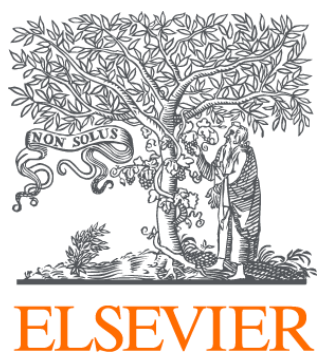

Since January 2020 Elsevier has created a COVID-19 resource centre with free information in English and Mandarin on the novel coronavirus COVID-

19. The COVID-19 resource centre is hosted on Elsevier Connect, the company's public news and information website.

Elsevier hereby grants permission to make all its COVID-19-related research that is available on the COVID-19 resource centre - including this research content - immediately available in PubMed Central and other publicly funded repositories, such as the WHO COVID database with rights for unrestricted research re-use and analyses in any form or by any means with acknowledgement of the original source. These permissions are granted for free by Elsevier for as long as the COVID-19 resource centre remains active. 


\title{
Comparison of vaccine-induced thrombotic events between ChAdOx1 nCoV-19 and Ad26.COV.2.S vaccines
}

\author{
Jimin Hwang ${ }^{\mathrm{a}, 1}$, Se Bee Lee ${ }^{\mathrm{b}, 1}$, Seung Won Lee ${ }^{\mathrm{c}, 1}$, Min Ho Lee ${ }^{\mathrm{d}}$, Ai Koyanagi ${ }^{\text {e,f }}$, \\ Louis Jacob ${ }^{\mathrm{e}, \mathrm{g}, \mathrm{h}}$, Kalthoum Tizaoui ${ }^{\mathrm{i}}$, Dong Keon Yon ${ }^{\mathrm{j},{ }^{, * *}}$, Jae Il Shin ${ }^{\mathrm{k}, *}$, Lee Smith ${ }^{1}$

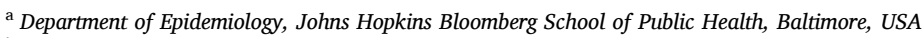 \\ ${ }^{\mathrm{b}}$ Ulsan University College of Medicine, Seoul, Republic of Korea \\ ${ }^{\mathrm{c}}$ Department of Data Science, Sejong University College of Software Convergence, Seoul, South Korea \\ ${ }^{\mathrm{d}}$ Yonsei University College of Medicine, Seoul, Republic of Korea \\ e Parc Sanitari Sant Joan de Deu/CIBERSAM, Universitat de Barcelona, Fundacio Sant Joan de Deu, Sant Boi de Llobregat, Barcelona, Spain \\ ${ }^{\mathrm{f}}$ ICREA, Pg. Lluis Companys 23, Barcelona, Spain \\ ${ }^{g}$ Centro de Investigación Biomédica en Red de Salud Mental (CIBERSAM), Madrid, Spain \\ ${ }^{\mathrm{h}}$ Faculty of Medicine, University of Versailles Saint-Quentin-en-Yvelines, Montigny-le-Bretonneux, France \\ ${ }^{\mathrm{i}}$ Laboratory of Microoranismes and Active Biomolecules, Sciences Faculty of Tunis, Tunis El Manar University, Tunis, Tunisia \\ ${ }^{\mathrm{j}}$ Department of Pediatrics, Seoul National University Hospital, Seoul National University College of Medicine, Seoul, South Korea \\ ${ }^{\mathrm{k}}$ Department of Pediatrics, Yonsei University College of Medicine, Seoul, Republic of Korea \\ ${ }^{1}$ The Cambridge Centre for Sport and Exercise Sciences, Anglia Ruskin University, Cambridge, UK
}

\section{A R T I C L E I N F O}

\section{Keywords:}

Vaccine-induced thrombotic thrombocytopenia

Thrombotic thrombocytopenia syndrome

Adenoviral vector vaccine

COVID-19 vaccine

Cerebral venous thrombosis

\begin{abstract}
A B S T R A C T
Cerebral venous thrombosis (CVT) events have been reported after vaccination with adenoviral COVID-19 vector vaccines. This study aimed to compare the clinical presentations and courses of vaccine-induced thrombotic thrombocytopenia (VITT) between the two adenoviral vector vaccines, Ad26.COV.2.S (Janssen/Johnson \& Johnson) and ChAdOx1 nCoV-19 (Astra-Zeneca). We found that CVT after Ad26.COV.2.S vaccination presents later with similar symptoms compared to CVT after administration of ChAdOx1 nCoV-19, albeit with more thrombosis and intracerebral hemorrhage, lower D-dimer and aPTT levels but similar mortality. These findings could help guide clinical assessment and management of CVT after COVID-19 vaccination.
\end{abstract}

\section{Introduction}

Reports of rare thrombotic events such as cerebral venous thromboses (CVT) have been accumulating after COVID-19 vaccination [1-5]. Severe vaccine-induced thrombotic thrombocytopenia (VITT) following vaccine administration was initially reported after ChAdOx1 nCoV-19 vaccination (Astra-Zeneca) [1-4], causing the European Medicine Agency (EMA) to issue a report on March 18, 2021 [6]. In the meantime, reports of CVT after another adenoviral vector-based vaccine, the Ad26. COV.2.S vaccine (Janssen/Johnson \& Johnson), resulted in the US Food and Drug Administration (FDA) recommending a pause in the use of the Ad26.COV.2.S vaccine as well [7]. Although the clinical course of VITT has been postulated to be similar to autoimmune heparin-induced thrombocytopenia $[1,2,5]$, the exact pathogenesis of VITT has not been fully elucidated, nor whether there are any differences in VITT events after the two adenoviral vector-based vaccines. Therefore, this study aimed to perform a rapid review to compare the clinical presentations of CVT cases between Ad26.COV.2.S and ChAdOx1 nCoV-19 vaccines.

\section{Methods}

\subsection{Study design and data source}

We conducted a rapid review to capture case reports of VITT after adenoviral vector-based COVID vaccine administration. We searched

\footnotetext{
* Corresponding author. 50 Yonsei-ro, Seodaemun-gu, C.P.O. Box 8044, Department of Pediatrics, Yonsei University College of Medicine, Seoul, 120-752, Republic of Korea.

** Corresponding author. FACAAI, Department of Pediatrics, Seoul National University College of Medicine, 103 Daehak-ro, Jongno-gu, Seoul, 03080, South Korea.

E-mail addresses: yonkkang@gmail.com (D.K. Yon), shinji@yuhs.ac (J.I. Shin).

${ }^{1}$ Co-first authors.
} 
PubMed ePubs, Scopus, Embase, and Web of Science databases to include articles published up to April 28, 2021, and related to COVID-19 vaccine-associated CVT after adenoviral vector-based COVID-19 vaccine administration. Case series with more than five cases were considered. We only included cases with CVT documented by clinical and radiologic findings after vaccination with the ChAdOx1 nCoV-19 or Ad26.COV.2.S vaccine. After reviewing individual abstracts and full texts, we identified 5 case series that met the inclusion criteria for this systematic review [1-5]. Laboratory measurements presented were either those obtained at time of admission or peak/nadir values.

\subsection{Statistical analysis}

Basic demographic and clinical information were presented as the median and interquartile range (IQR) for continuous variables and the percentage for categorical variables. Variables were compared with the Mann-Whitney U test for continuous variables and Fisher's exact test for categorical variables. Statistical significance was defined as two-tailed $\mathrm{p}$ $<0.05$. Statistical analyses were performed with $\mathrm{R}$ version 4.0.4 ( $\mathrm{R}$ Core Team, Vienna, Austria).

\section{Results}

Demographic and clinical characteristics of 40 patients (ChAdOx1 $\mathrm{nCoV}-19, \mathrm{n}=28$; Ad26.COV.2.S, $\mathrm{n}=12$ ) are shown in Table 1 and Supplementary Table S1. Patients who received the Ad26.COV.2.S vaccine tended to have clinical manifestations later than those administered ChAdOx1 nCoV-19, with a median of 16 days post-vaccination compared to 10 days $(\mathrm{p}=0.004)$. D-dimer levels were significantly lower in the Ad26.COV.2.S group (16.3 vs. 74.2 times the upper limit of normal, $\mathrm{p}=0.036$ ), and aPTT levels were also lower in the Ad26.COV.2. $S$ group with borderline significance ( $28.0 \mathrm{vs.} 34.0 \mathrm{~s}, \mathrm{p}=0.086)$. Other clinical characteristics were similar, with no significant differences in age or sex distribution, platelet counts, prothrombin time, fibrinogen levels. In both groups, almost all patients had positive tests for HIT antibody enzyme-linked immunosorbent assay (ELISA) $(96.0 \%$ vs. $100.0 \%, p=1.000$ ). However, while $92.9 \%$ of patients administered ChAdOx1 nCoV-19 had positive platelet function tests, only $11.1 \%$ had positive tests in the Ad26.COV.2.S group ( $\mathrm{p}<0.001$ ).

Interestingly, patients with CVT after Ad26.COV.2.S administration

Table 1

Different clinical characteristics of patients with CVT after COVID-19 vaccination according to vaccine type.

\begin{tabular}{|c|c|c|c|c|}
\hline \multirow[t]{2}{*}{ Characteristic } & \multirow{2}{*}{$\begin{array}{l}\text { Total }<(\mathrm{n}=40) \\
\text { Number of } \\
\text { patients } \\
(\%) /<\text { Median } \\
{[\mathrm{IQR}]}\end{array}$} & \multirow{2}{*}{$\begin{array}{l}\text { ChAdOx1 } \\
\text { nCoV-19/Astra- } \\
\text { Zeneca }(\mathrm{n}=28) \\
\text { Number of } \\
\text { patients } \\
(\%) /<\text { Median } \\
{[\text { IQR }]}\end{array}$} & \multirow{2}{*}{ 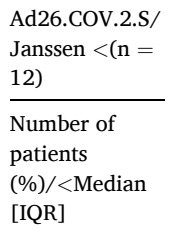 } & \multirow[b]{2}{*}{$\begin{array}{l}\mathrm{P} \text { - } \\
\text { value }\end{array}$} \\
\hline & & & & \\
\hline $\begin{array}{l}\text { Days to } \\
\text { admission }\end{array}$ & $11.0[9.0 ; 16.0]$ & $10.0[8.0 ; 13.5]$ & $\begin{array}{l}16.0[12.0 ; \\
18.0]\end{array}$ & 0.004 \\
\hline $\begin{array}{l}\text { D-dimer (ratio } \\
\text { to upper } \\
\text { limit of } \\
\text { normal } \\
\text { range) }\end{array}$ & $\begin{array}{l}52.0 \text { [15.7; } \\
124.7]\end{array}$ & $\begin{array}{l}74.2[32.6 ; \\
140.0]\end{array}$ & $\begin{array}{l}16.3[12.2 ; \\
61.7]\end{array}$ & 0.036 \\
\hline $\begin{array}{l}\text { Functional HIT } \\
\text { assay } \\
\text { positive }\end{array}$ & $14 / 23(60.9 \%)$ & $13 / 14(92.9 \%)$ & 1/9 (11.1\%) & $\begin{array}{l}< \\
0.001\end{array}$ \\
\hline $\begin{array}{l}\text { Presence of } \\
\text { intracerebral } \\
\text { hemorrhage }\end{array}$ & $12 / 40(30.0 \%)$ & $5 / 28(17.9 \%)$ & 7/12 (58.3\%) & 0.021 \\
\hline $\begin{array}{l}\text { Internal } \\
\text { jugular vein } \\
\text { thrombosis }\end{array}$ & $7(12.3 \%)$ & $1(2.2 \%)$ & $6(50.0 \%)$ & $\begin{array}{l}< \\
0.001\end{array}$ \\
\hline
\end{tabular}

IQR: interquartile range; HIT: heparin-induced thrombocytopenia. were more likely to also have intracerebral hemorrhage $(58.3 \%$ vs. $17.9 \%, \mathrm{p}=0.021$ ). They were also more likely to have additional thrombosis other than CVT, with borderline significance $(66.7 \%$ vs. $32.1 \%, \mathrm{p}=0.063$ ). Notably, patients with CVT after Ad26.COV.2.S administration were more likely to also have internal jugular vein (IJV) thrombosis $(2.2 \%$ vs. $50 \%, \mathrm{p}<0.001)$. However, there was no statistically significant difference in mortality $(25.0 \%$ vs. $56.5 \%, \mathrm{p}=0.152)$. There were no significant differences in presenting symptoms, most commonly being headache, visual disturbance, hemiparesis, and fever.

\section{Discussion}

The current study is the first to directly compare CVT profiles after COVID-19 vaccine administration between the two adenoviral vectorbased vaccines, Ad26.COV.2.S and ChAdOx1 nCoV-19. Although the main presenting symptoms were similar, with the majority reporting headaches and neurologic manifestations, patients who received Ad26. COV.2.S tended to present CVT later, with a median time to admission of 16 days post-vaccination compared to 10 days after ChAdOx1 nCoV-19. Therefore, it is essential to educate patients to be vigilant for neurologic symptoms for a longer period after Ad26.COV.2 vaccination.

Patients with CVT after the Ad26.COV.2.S vaccine had lower Ddimer and aPTT levels, even though there was a greater likelihood of other thrombosis or intracerebral hemorrhage in this group. From the findings of our study, it can be inferred that CVT events after the Ad26. COV.2.S vaccine, even with a seemingly adverse radiologic profile, has a milder, more insidious clinical course. This could be due to the differential immunogenic profiles of ChAdOx1 nCoV-19 and Ad26.COV.2.S vaccines in stimulating interferon-gamma levels, which is a known mediator in diffuse intravascular coagulation [8-10].

Even though the mortality of patients with CVT after Ad26.COV.2.S administration was $25.0 \%$, lower than the $56.5 \%$ from cases after ChAdOx1 nCoV-19, there was no statistically significant difference. However, as the sample size was relatively small, additional data for higher statistical power is required to better assess differences in mortality.

We found that anti-PF4/heparin antibodies were overwhelmingly present in most patients with either vaccine, whereas the platelet function assay results were strikingly different between the Ad26. COV.2.S and ChAdOx1 nCoV-19 vaccines. Typically, anti-PF4/heparin ELISA is regarded as a sensitive screening tool, but platelet function assays are the gold standard for the diagnosis of heparin-induced thrombocytopenia [11,12]. The discrepancy of anti-PF4/heparin ELISA and platelet function tests adds a question mark regarding the previously hypothesized heparin-induced thrombocytopenia -like mechanism. The results also need to be interpreted with caution, however, as platelet function tests tend to vary by testing center, and different testing methods were used between studies [1-3,5].

There are some limitations to our study. First, the sample size was small, considering the relatively recent, rare nature of VITT. Second, cases of CVT after Ad26.COV.2.S vaccination were based in the United States, whereas cases associated with ChAdOx1 $\mathrm{nCoV}-19$ were all based in Europe, which may have contributed to differences in management. Lastly, as this study was a systematic review of case reports, the timing of laboratory measurements differed between studies. Therefore, it should be stated that our study is intended to provide descriptive information for future, more definitive studies, and not to confirm any hypothesis. However, understanding these different characteristics between vaccines could help guide further investigations and clinical management as both Ad26.COV.2.S and ChAdOx1 nCoV-19 vaccines are being distributed worldwide.

\section{Conclusions}

This study found that CVT after Ad26.COV.2.S vaccination presents later with similar symptoms compared to CVT after administration of 
ChAdOx1 nCoV-19, albeit with more thrombosis and intracerebral hemorrhage, lower D-dimer and aPTT levels but similar mortality. Understanding different characteristics between vaccines could help guide future clinical management of VITT.

\section{Author statement}

Jimin Hwang: Conceptualization, Formal analysis, Writing - original draft, Writing - review \& editing. Se Bee Lee: Data curation, Writing original draft, Writing - review \& editing. Seung Won Lee: Writing original draft, Writing - review \& editing. Min Ho Lee: Writing - original draft, Writing - review \& editing. Ai Koyanagi: Writing - original draft, Writing - review \& editing. Louis Jacob: Writing - original draft, Writing - review \& editing. Kalthoum Tizaoui: Writing - original draft, Writing review \& editing. Dong Keon Yon: Supervision, Writing - original draft, Writing - review \& editing. Jae Il Shin: Conceptualization, Supervision, Writing - original draft, Writing - review \& editing. Lee Smith: Writing original draft, Writing - review \& editing.

\section{Funding statement}

This research did not receive any specific grant from funding agencies in the public, commercial, or not-for-profit sector.

\section{Declaration of competing interest}

None.

\section{Acknowledgments}

We thank Elena Dragioti and Joaquim Radua for their valuable discussions and feedback.

\section{Appendix A. Supplementary data}

Supplementary data to this article can be found online at https://doi. org/10.1016/j.jaut.2021.102681.

\section{References}

[1] A. Greinacher, T. Thiele, T.E. Warkentin, K. Weisser, P.A. Kyrle, S. Eichinger, Thrombotic thrombocytopenia after ChAdOx1 nCov-19 vaccination, N. Engl. J. Med. (April 9, 2021), https://doi.org/10.1056/NEJMoa2104840. Published online.

[2] M. Scully, D. Singh, R. Lown, et al., Pathologic antibodies to platelet factor 4 after ChAdOx1 nCoV-19 vaccination, N. Engl. J. Med. (April 16, 2021), https://doi.org/ 10.1056/NEJMoa2105385. Published online.

[3] N.H. Schultz, I.H. Sørvoll, A.E. Michelsen, et al., Thrombosis and thrombocytopenia after ChAdOx1 nCoV-19 vaccination, N. Engl. J. Med. (April 9, 2021), https://doi.org/10.1056/NEJMoa2104882. Published online.

[4] A. Tiede, U.J. Sachs, A. Czwalinna, et al., Prothrombotic Immune Thrombocytopenia after COVID-19 Vaccine, Blood, April 28, 2021, https://doi. org/10.1182/blood.2021011958. Published online.

[5] I. See, J.R. Su, A. Lale, et al., US Case Reports of Cerebral Venous Sinus Thrombosis with Thrombocytopenia after Ad26.COV2.S Vaccination, March 2 to April 21, 2021, JAMA, April 30, 2021, https://doi.org/10.1001/jama.2021.7517. Published online.

[6] European Medicines Agency, AstraZeneca's COVID-19 Vaccine: EMA Finds Possible Link to Very Rare Cases of Unusual Blood Clots with Low Platelets, European Medicines Agency, April 6, 2021. Published, https://www.ema.europa. eu/en/news/astrazenecas-covid-19-vaccine-ema-finds-possible-link-very-rare-case s-unusual-blood-clots-low-blood. (Accessed 29 April 2021).

[7] Commissioner O of the, Joint CDC and FDA Statement on Johnson \& Johnson COVID-19 Vaccine, FDA, April 13, 2021. Published, https://www.fda.gov/news -events/press-announcements/joint-cdc-and-fda-statement-johnson-johns on-covid-19-vaccine. (Accessed 14 May 2021).

[8] K.J. Ewer, J.R. Barrett, S. Belij-Rammerstorfer, et al., T cell and antibody responses induced by a single dose of ChAdOx1 nCoV-19 (AZD1222) vaccine in a phase $1 / 2$ clinical trial, Nat. Med. 27 (2) (2021) 270-278, https://doi.org/10.1038/s41591020-01194-5.

[9] K.E. Stephenson, M. Le Gars, J. Sadoff, et al., Immunogenicity of the Ad26.COV2.S vaccine for COVID-19, J. Am. Med. Assoc. 325 (15) (2021) 1535-1544, https://doi. org/10.1001/jama.2021.3645.

[10] Y. Shinozawa, X.Q. Xie, T. Endo, K. Takuma, K. Koike, [Correlation between intravascular coagulation/fibrinolysis system and cytokines], Nihon Rinsho 62 (12) (2004) 2253-2261.

[11] V. Minet, J.-M. Dogné, F. Mullier, Functional assays in the diagnosis of heparininduced thrombocytopenia: a review, Molecules 22 (4) (2017), https://doi.org/ 10.3390/molecules22040617.

[12] K.K. Sahu, V. Jindal, J. Anderson, A.D. Siddiqui, I.A. Jaiyesimi, Current perspectives on diagnostic assays and anti-PF4 antibodies for the diagnosis of heparin-induced thrombocytopenia, Hematol. Res. Rev. 11 (2020) 267-277, https://doi.org/10.2147/JBM.S232648. 